\title{
Ciencia Abierta y su papel durante la pandemia de COVID-19
}

\author{
Omar Díaz Fragoso* \\ Gabriela María Luisa Riquelme Alcantar* \\ Gibrán Rivera González ${ }^{\star *}$
}

Artículo recibido:

5 de febrero de 2021

Artículo aceptado:

2 de marzo de 2021

Artículo de investigación

\section{RESUMEN}

El objetivo de esta investigación es analizar el papel de la Ciencia Abierta en la pandemia de COVID-19 con el fin de entender el uso que dicho modelo exhibe en el contexto de la emergencia sanitaria. El diseño metodológico incluyó la selección y el análisis de 43 iniciativas de Ciencia Abierta que buscan brindar soluciones para enfrentar la pandemia. De dichas iniciativas se revisó el objetivo, el alcance, los actores participantes, los beneficios y los productos asociados con su implementación. Los resultados permiten constatar que el modelo de Ciencia Abierta se ha empleado para 1)

* Instituto Politécnico Nacional (IPN), Centro de Investigaciones Económicas, Administrativas y Sociales (CIECAS), México omar.dz.21@gmail.com griquelme@ipn.mx

** Instituto Politécnico Nacional (IPN), Unidad Profesional Interdisciplinaria de Ingeniería y Ciencias Sociales y Administrativas (UPIICSA), Méxicoｇriverag@ipn.mx

INVESTIGACIÓN BIBLIOTECOLÓGICA, vol. 35, núm. 88, julio/septiembre, 2021, México, ISSN: 2448-8321 pp. 147-160 
educar e informar a la sociedad, 2) proveer recursos a la comunidad científica para realizar investigaciones, y 3) suministrar equipo médico, equipo de protección y vacunas. Asimismo, se constata que el modelo ofrece beneficios para la comunidad científica y la sociedad; no obstante, existen ciertos aspectos que, en la crisis sanitaria, se revelan como áreas de oportunidad. En conclusión, el desarrollo de iniciativas de Ciencia Abierta durante la pandemia por el virus SARS-CoV-2 es aleccionador pues su implementación permite presenciar las posibilidades que proporciona para facilitar el flujo de información, desarrollar el conocimiento científico y buscar soluciones en momentos difíciles para la humanidad.

Palabras clave: Ciencia Abierta; Iniciativas; COVID-19; SARS-CoV-2

Open Science and its role during the COVID-19 pandemic Omar Díaz Fragoso, Gabriela María Luisa Riquelme Alcantar and Gibrán Rivera González

\section{Abstract}

The aim of this study is to analyze the role of Open Science in the COVID-19 pandemic to understand the use that this model exhibits in the context of the health emergency. The methodological design included the selection and analysis of 43 Open Science initiatives that seek to provide solutions to face the pandemic. The results show that the Open Science model has been used to 1 . educate and inform the society, 2 . provide resources to the scientific community to conduct research and 3. supply medical equipment, personal protection equipment and vaccines. Likewise, it is found that the model offers benefits for the scientific community and society, however, there are certain aspects that, in the health crisis, are revealed as areas of opportunity. In conclusion, the development of Open Science initiatives during the SARS-CoV-2 virus pandemic is instructive because its implementation allows us to witness the possibilities it provides to facilitate the flow of information, develop scientific knowledge, and seek solutions in difficult times for humanity.

Keywords: Open Science; Initiatives; COVID-19; SARS-CoV-2 


\section{INTRODUCCIÓN}

T a pandemia generada por el virus SARS-CoV-2 ha provocado una crisis ـmundial con efectos adversos para la humanidad. Más de dos millones de vidas se han perdido en el mundo y los contagios aumentaron de manera exponencial entre febrero y diciembre de 2020 (https://coronaviruswatch.ircai.org/). En consecuencia, los sistemas de salud de algunas naciones se han visto rebasados en la atención de personas contagiadas (Shadmi et al., 2020; Hick y Biddinger, 2020; Armocida et al., 2020) Más aún, las medidas de confinamiento impuestas por ciertos gobiernos provocaron el cierre de establecimientos cuya permanencia se volvió insostenible con la consabida pérdida de empleos (Taskinsoy, 2020; Joshi, Bhaskar y Gupta, 2020). Según el Banco Mundial, la crisis de salud hundió la economía global en la peor recesión desde la Segunda Guerra Mundial (World Bank, 2020). En el caso de los países emergentes y en vías de desarrollo, los estragos provocados por el virus serán más notorios (ILO, 2020; World Bank, 2020). Sin duda, la crisis obliga a repensar y replantear diversos aspectos de la vida en sociedad; entre ellos y de particular relevancia para este artículo, el papel de la ciencia.

La emergencia sanitaria requiere la movilización de distintos actores de la sociedad a fin de encontrar soluciones viables a la problemática. En este sentido, la institución científica es uno de los actores principales en la búsqueda de alternativas. De acuerdo con Olivé (2007: 38), la ciencia es uno de los bienes públicos "que pueden ser utilizados para aumentar el bienestar social y para resolver una diversidad de problemas". En el contexto de la pandemia, ésta puede coadyuvar en el desarrollo de vacunas, en la experimentación de tratamientos médicos, en el diseño de ventiladores y equipo de protección personal, así como en la comprensión de los fenómenos sociales asociados con la situación actual. Sin embargo, para que todos estos procesos ocurran eficientemente se requiere un modelo que promueva y favorezca la colaboración entre científicos, universidades, centros de investigación, grupos editoriales, gobiernos, instituciones sanitarias, personal médico, industria y ciudadanía.

Ante este panorama, la Ciencia Abierta (en adelante, CA) aspira a contribuir en la búsqueda de la colaboración deseada a fin de brindar soluciones ante la pandemia; constituye un modo innovador de producción social que, con base en la digitalización y la conectividad, busca abrir las etapas de la investigación científica a fin de compartir los procesos, recursos y resultados de las investigaciones financiadas con recursos públicos (García-Peñalvo, 2010; Peters, 2010; OECD, 2015). En efecto, la CA contempla la apertura de publicaciones, datos de investigación, códigos, apuntes, métodos, revisión 
por pares y recursos educativos (Vicente-Saez y Martinez-Fuentes, 2018; McKiernan et al., 2016). Su fin último es que el conocimiento se distribuya de forma gratuita, transparente y reutilizable para acelerar el progreso científico (Hampton et al., 2015; García-Peñalvo, 2010) en beneficio de la humanidad. De hecho, Vicente-Saez y Martinez-Fuentes (2018) señalan que además de la comunidad científica, las investigaciones podrían ser aprovechadas por actores políticos, empresariales y ciudadanos.

Si bien existen iniciativas que tienen como objetivo la apertura de la ciencia, la crisis por la pandemia se revela como una coyuntura definitoria. En la última década, naciones como Estados Unidos y el Reino Unido establecieron lineamientos que exigen compartir los elementos de las investigaciones financiadas con recursos públicos (NSF, 2015; HEFCE et al., 2016). De igual forma, miembros de la Unión Europea suscribieron el programa Horizon 2020 cuya intención es estimular con financiamiento el desarrollo científico y tecnológico para promover la innovación, así como la circulación del conocimiento (EC, 2011). Éste estipulaba que, para 2020, las publicaciones y, en algunos casos, los datos de las investigaciones financiadas por el programa deben ser de libre acceso. Ahora bien, la pandemia provocó la proliferación de distintas iniciativas que persiguen la diseminación del conocimiento a fin de acelerar la búsqueda de soluciones ante el problema que enfrenta la sociedad (Mandal, 2020; Zastrow, 2020). Sin embargo, es escasa la reflexión en torno al impacto que ha tenido el súbito desarrollo de iniciativas de CA en la crisis actual. Por lo anterior, el objetivo de este artículo es analizar el papel de la CA en la pandemia de COVID-19 a fin de entender el uso que se le ha dado a dicho modelo en el contexto de la crisis sanitaria.

\section{Metodología}

Es un estudio cualitativo centrado en un muestreo deliberado pues se recolectaron datos que permitieron atender el objetivo de la investigación (White y Marsh, 2006). En total, se revisaron 43 iniciativas que buscan brindar soluciones a la crisis sanitaria de SARS-CoV-2. Éstas se obtuvieron de "Open access to facilitate research and information on COVID-19", documento publicado por la Organización de las Naciones Unidas para la Educación, la Ciencia y la Cultura (Unesco, por sus siglas en inglés) que contiene un extenso listado de recursos y contempla diversos modos de CA (Unesco, 2020b). Esta información se complementó con la obtenida de la Organización para la Cooperación y el Desarrollo Económicos (OECD, por sus siglas en inglés) (OECD. Secretary-General, 2020). Su elección se debe a que tanto la Unesco como la OECD 
son organismos reconocidos internacionalmente por promover el libre acceso a la información científica (Unesco, 2017b; 2017a; OECD, 2015; 2007).

En cada una de las iniciativas se analizó 1) el objetivo: propósito que se desea alcanzar; 2) el alcance: el área territorial que se cubre; 3) los actores participantes: individuos, instituciones y organizaciones que colaboran en la implementación de las iniciativas; 4) los beneficios: asociados con los efectos útiles y positivos que se brindan y, finalmente, 5) los productos: bienes tangibles que generan las iniciativas. Para conservar el rastro de las ideas y decisiones que emergieron durante el proceso se redactaron un conjunto de memorándums, mismos que permitieron vincular la recolección de datos con la escritura de los resultados de la investigación (Charmaz, 2006).

\section{PRESENTACIÓN Y ANÁlisis DE RESUltados}

En el contexto de la pandemia, el modelo de CA se ha utilizado con el fin de mitigar el virus SARS-CoV-2. El análisis de los datos asociados con las 43 iniciativas revisadas permitió identificar tres usos que se le han dado al modelo. Primero, para educar e informar a la sociedad con base en información actualizada sobre el desarrollo de la pandemia, contenido informativo para aclarar dudas y Recursos Educativos Abiertos. Segundo, la CA provee recursos a la comunidad cientifica para realizar investigaciones. Lo anterior es posible con el libre acceso a publicaciones científicas, datos de investigación, modelos matemáticos, simulaciones, capacidades computacionales y con el involucramiento de la ciudadanía. Tercero, simplificar el suministro de equipo médico, de protección y de vacunas por medio de iniciativas que ponen a disposición patentes, códigos para manufacturar equipo médico, así como estrategias para asegurar un acceso igualitario a vacunas. Enseguida, se profundiza sobre cada uno de estos usos y se mencionan ciertas iniciativas representativas.

Un primer uso que tiene la CA en la pandemia es educar e informar a la sociedad. Existen iniciativas como Coronavirus Watch (https://coronaviruswatch.ircai.org/) y Johns Hopkins Coronavirus Resource Center (https://coronavirus.jhu.edu/) que tienen el objetivo de proporcionar una visión global del estado de la pandemia. ${ }^{1}$ En una pantalla principal, éstas brindan información constantemente actualizada sobre el número de casos positivos, fallecimientos, personas recuperadas, pruebas y vacunas aplicadas. En adición, despliegan notas periodísticas que permiten estar al día sobre el tema. Por otra parte, hay iniciativas que buscan

1 Los sitios web que se mencionan de aquí en adelante fueron consultados entre el 18 y el 21 de diciembre de 2020. 
diseminar información entre la ciudadanía sobre cómo evitar contagios, qué hacer en caso de estar contagiado, cómo identificar un posible caso positivo, qué tipo de enfermedad es la COVID-19, así como la clarificación de mitos en torno al padecimiento. Un par de ejemplos son AfricArXiv COVID-19 (https://info.africarxiv.org/covid-19/) y \#DontGoViral (http://www. dontgoviral.wiki/index.php/Welcome). El primero, además de brindar acceso a información científica, ofrece videos, emisiones de podcasts y un chat automatizado que orientan al usuario sobre el padecimiento. El segundo trata de evitar la desinformación al convocar a artistas a crear canciones, pinturas, poesía o cortometrajes que transmiten información de salud pública con un lenguaje cotidiano. Más aún, ofrecen información en distintas lenguas con el fin de que sea accesible para cualquiera.

La diseminación de la información en el contexto de la CA debe llegar a la mayor cantidad de ciudadanos, incluso a los hablantes de lenguas indígenas. Por ello, la Unesco (2020a) encabeza la iniciativa COVID-19 Pandemic: Language Matters que oferta recursos de información sobre la COVID-19 en distintas lenguas. Por otro lado, la imposición del confinamiento obligó a cerrar escuelas y universidades. En consecuencia, se promovieron iniciativas como OER4Covid (https://oer4covid.oeru.org/) y Call for Joint Action: Supporting Learning and Knowledge Sharing through Open Educational Resources (OER) (Chakchouk y Giannini, 2020), que buscan concientizar sobre la importancia de adoptar recursos educativos abiertos, así como otorgar asesoría para educadores e instituciones que requieren migrar al aprendizaje en línea. No obstante, su adopción genera inquietud sobre la infracción de los derechos de autor del contenido utilizado. Por lo anterior, un conjunto de especialistas en derechos de autor emitió el Public Statement of Library Copyright Specialists: Fair Use E Emergency Remote Teaching \& Research (Algenio et al., 2020) que clarifica la aplicación de las leyes en Estados Unidos durante la pandemia. Finalmente, educar e informar requiere de redes y servicios de banda ancha por lo que Agenda for Action: For Faster and Better Recovery (Broadband Commission for Sustainable Development, 2020) ejecuta acciones para fortalecer la infraestructura, otorgar facilidades de conexión, acceder a información de calidad y brindar seguridad cibernética a los usuarios.

Asimismo, la CA ha mostrado ser de utilidad para proveer recursos a la comunidad cientifica para realizar investigaciones durante la crisis. Asesores nacionales de ciencia y tecnología de distintos países emitieron la iniciativa Call for Open Access to COVID-19 Publications (https://www.ic.gc.ca/eic/site/063. nsf/eng/h_98016.html) con el objetivo de hacer un llamado a los grupos editoriales para que pongan a disposición de los investigadores y del público las publicaciones científicas sobre el virus SARS-CoV-2. En consecuencia, algunos grupos como Elsevier, Emerald Publishing, SAGE Publishing, Springer 
Nature y Wiley permiten acceder libremente a artículos científicos relacionados con la pandemia. En efecto, éstos en colaboración con otros actores promueven la Public Health Emergency COVID-19 Initiative (NCBI y NLM, 2020) que posibilita que los grupos editoriales den acceso inmediato a las publicaciones sobre la pandemia en el archivo de ciencias biomédicas y de la vida PubMed Central. Por otra parte, en Brasil y China se desarrollaron plataformas como el Directorio de fuentes de información cientifica de acceso abierto sobre Coronavirus (Ibict, 2020) y la Scientific Literature Sharing Platform Built for COVID-19 Studies (CAS, 2020) que brindan acceso a artículos derivados de investigaciones locales e internacionales.

Además de la apertura de artículos científicos, también se comparten los datos asociados con las investigaciones sobre la pandemia. En este sentido, posicionamientos como "Sharing research data and findings relevant to the novel coronavirus (COVID-19) outbreak" (Wellcome, 2020) exhortan a científicos, financiadores y revistas a compartir libremente datos que coadyuven en los esfuerzos para vencer al virus SARS-CoV-2 y salvar vidas. En consecuencia, se han establecido esfuerzos como RDA-COVID19 (RDA, 2020) que busca definir lineamientos claros y detallados para diseminar datos de investigación en las circunstancias de la pandemia, teniendo en cuenta los intereses de los actores involucrados. Más aún, se desea que los datos distribuidos cumplan con los principios FAIR para que sean localizables, accesibles, interoperables y reutilizables. También, existen proyectos como COVID-19 Data Portal (https://www.covid19dataportal.org) y OpenAIRE for COVID-19 (https://www.openaire.eu/openaire-activities-for-covid-19) que permiten a los investigadores cargar, acceder y analizar datos de referencia acerca del virus y tópicos relacionados. Asimismo, se constatan iniciativas como Vivli (https://search.vivli.org/) que, en el contexto de la crisis, permite compartir y acceder sin costo a datos anonimizados de ensayos clínicos sobre el virus. En fin, estas iniciativas y otras dan oportunidad a los científicos de servirse de secuencias virales, expresiones genéticas, proteínas, bioquímica, imágenes de microscopio, entre otros.

Por otro lado, la CA facilita la distribución, así como la reutilización de modelos matemáticos y de simulaciones. Iniciativas como MOBS Lab (2020) y MIDAS (2020) se enfocan en el desarrollo de éstos, así como de herramientas computacionales para mejorar la comprensión de la patogénesis, la transmisión, las estrategias de control y previsión en torno al virus. Justamente, proporcionan la posibilidad de recibir formación en webinars, solicitar recursos para proyectos y acceder libremente a modelos, simulaciones y datos. The COVID-19 High Performance Computing Consortium (https://covid19-hpc-consortium.org/) cuenta con la participación 
de la academia, gobierno e industria y brinda capacidades computacionales, asesoría y servicios para los proyectos que requieren ejecutar programas complejos. Adicionalmente, durante la pandemia se ha promovido la Ciencia Ciudadana. Un ejemplo es Folding@bome (https://foldingathome. org/diseases/infectious-diseases/covid-19/), que pretende comprender cómo funcionan las proteínas virales del SARS-CoV-2 y diseñar terapias para detenerlas. Para lograrlo, además de dar libre acceso a datos y algoritmos, permite que la ciudadanía desde su hogar ponga a disposición de la iniciativa las capacidades de sus equipos de cómputo con el fin de acelerar la ejecución de simulaciones. Asimismo, existen juegos como Foldit (https:// fold.it/portal/) que involucran a la ciudadanía en la manipulación de proteínas para conocer su estructura y diseño. Mientras el jugador progresa, se generan datos que mejoran el entendimiento de la comunidad científica sobre las proteínas y el combate de enfermedades.

En el mismo sentido, el tercer uso de la CA posibilita el suministro de equipo médico, de protección y de vacunas. Open COVID Pledge (https:// opencovidpledge.org/the-pledge/) es una iniciativa que tiene el objetivo de dar libre acceso a propiedad intelectual para que se utilice con el fin de erradicar la pandemia de la COVID-19 y minimizar el impacto de la enfermedad. Ésta es encabezada por Creative Commons y cuenta con la participación de la comunidad científica, empresas y abogados. En su portal ofrece orientación sobre las licencias existentes para aquellos que desean distribuir sus patentes y derechos de autor, así como acceso a propiedad intelectual que distintas empresas e inventores desarrollaron para combatir el virus. Otro caso es el de Tarek Loubani y el proyecto Glia, que cuenta con el apoyo de la beca Bassel Khartabil (Bassel Khartabil Fellowship, 2020). Éstos dan acceso a planes abiertos de equipo médico, por lo que cualquier persona con impresora 3D puede producir equipos a bajo costo. Entre los equipos desarrollados están protectores faciales, estetoscopios, torniquetes y otoscopios. Finalmente, la iniciativa COVAX (CEPI, 2020) tiene como meta asegurar acceso igualitario a las vacunas contra la COVID-19. Para ello ofrece financiamiento para el desarrollo de vacunas, gestión y monitoreo del portafolio de proyectos, así como asesoría en áreas críticas del desarrollo. Cabe señalar que en ésta participan organizaciones públicas, privadas, filantrópicas y de la sociedad civil.

\section{Discusión}

Los resultados del estudio revelan que los usos de la CA durante la pandemia brindan beneficios. De acuerdo con Choudhury et al. (2014), la aplicación 
del modelo acelera el progreso científico pues se construye conocimiento a partir de información existente. En este caso, la apertura de publicaciones y datos sobre el virus ha facilitado que la comunidad científica adquiriera conocimiento sobre la evolución de éste y genere nuevos hallazgos, así como soluciones para enfrentarlo. Un ejemplo son las vacunas, pues la información disponible ha sido utilizada para desarrollarlas en un breve plazo de tiempo (Doshi, 2020). De igual forma, lo que señalan Pfenninger et al. (2017) respecto al ahorro de tiempo y de recursos gracias a la apertura de los elementos de las investigaciones se refuerza en la situación actual. En efecto, además del rápido desarrollo de vacunas, algunas iniciativas posibilitan la reducción de tiempos y costos asociados con el diseño, fabricación y distribución de equipo médico, así como de protección personal; particularmente cuando existe escasez de éstos en el mundo.

El uso de la CA permite constatar la cooperación entre diversos actores. Según Whyte y Pryor (2011), su implementación genera colaboraciones entre los miembros de la comunidad científica. En el caso de la pandemia se observa que la gravedad de la situación detonó, aunado a la colaboración entre investigadores, la incorporación de gobiernos, empresas privadas, organismos internacionales, organizaciones de la sociedad civil y asociaciones de especialistas en esfuerzos encaminados a facilitar la actividad científica. Asimismo, se busca involucrar a la ciudadanía en los temas de ciencia relacionados con la COVID-19. Más aún, retomando las ideas de Elliott y Resnik (2019), se nota que la diseminación de información no es sólo relevante científicamente, pues también lo es en términos sociales. Primero, por aquellas iniciativas que pretenden informar a la ciudadanía acerca de la evolución de la pandemia, la naturaleza del padecimiento, medidas de prevención y mitigación en caso de poseer el virus. Segundo, por los proyectos que buscan asegurar la educación de los niños y jóvenes que no están en condiciones de regresar a las aulas. Finalmente, en ambos casos la evidencia muestra lo expuesto por Chapman y Greenhow (2019), quienes señalan que la CA sirve para informar y educar a la sociedad en la era digital.

Si bien la CA tiene efectos positivos, la pandemia ha permitido vislumbrar algunos riesgos. En este sentido, la urgencia por llegar a resultados que permitan reducir los estragos del virus SARS-CoV-2 provoca que se disemine información que no está científicamente verificada (Van Schalkwyk et al., 2020). Por ello, iniciativas como COVID-19 Data Portal y Public Health Emergency COVID-19 Initiative (NCBI y NLM, 2020), que dan libre acceso a artículos científicos o datos de investigación, cuentan con mecanismos para validar la información antes de compartirla en repositorios digitales. Aunque éstos no son infalibles, reducen el riesgo de publicar información falsa. Por otra parte, 
la manufactura en impresión 3D de equipo médico y de protección a partir de códigos abiertos pone de manifiesto que en algunos casos el equipo de protección que se fabrica no cumple con los requerimientos estipulados por la autoridad sanitaria (Zastrow, 2020). Por ello, quien los utiliza tiene una falsa sensación de seguridad pues no está contribuyendo a reducir la probabilidad de contagio. En fin, el uso de la CA en el contexto de una emergencia sanitaria debe analizarse de forma crítica para evitar resultados perniciosos.

\section{CONSIDERACiOnES FINALES}

La ciencia es un bien central en la búsqueda de soluciones a las problemáticas que afectan a la humanidad. Tradicionalmente, el acceso a ella ha permanecido al alcance de unos cuantos y deja de lado a gran parte de la sociedad. Sin embargo, el desarrollo tecnológico, así como la gravedad e incertidumbre de las dificultades que se manifiestan en la actualidad, obligaron a repensar los mecanismos de acción de la actividad científica. Por ello, desde hace algunos años se establecieron iniciativas para promover un modelo de CA. Si bien la pandemia por el virus SARS-CoV-2 es una desgracia para el mundo, su gravedad detonó una serie de circunstancias en las que la CA tiene posibilidades de demostrar su potencial. De hecho, se percibe una colaboración sin precedentes entre la academia, el gobierno, la industria y la sociedad civil.

Además, se debe reconocer que la aplicación del modelo revela distintas áreas de oportunidad, por lo que resulta imprescindible contar con estudios críticos y reflexivos que permitan conocer el uso de la CA en sus distintas modalidades; particularmente, en el contexto de emergencias sanitarias. $\mathrm{Pa}$ ra futuras investigaciones se sugiere que se evalúe el impacto que las iniciativas tuvieron en la lucha contra la COVID-19. Ciertamente, la pandemia es un momento clave que propicia transformar la manera de hacer ciencia. Su aplicación podría replicarse para enfrentar con la misma vehemencia otros brotes virales como los de ébola y zika. Sin duda, los usos de la CA durante la emergencia sanitaria sugieren un futuro prometedor para su desarrollo.

Agradecimientos

Se dan las gracias a la Secretaría de Investigación y Posgrado del Instituto Politécnico Nacional, quien auspició los proyectos de investigación 20195975, 20201625, 20195611 y 20200777 para la realización de este artículo de investigación. De igual forma, se agradece al Fondo FOSEC SEP-Investigación Básica del Conacyt el apoyo para financiar el proyecto "Ciencia abierta en México: Diagnóstico y estrategias para promover su cultura en las IES y 
Centros de Investigación del país”. Finalmente, se reconoce al Programa de Estímulos a los Investigadores del Instituto Politécnico Nacional, al Sistema Nacional de Investigadores, así como al Programa de Becas para Estudios de Posgrado del Conacyt.

\section{REFERENCIAS}

Algenio, Emilie, Sara Benson, Josh Bolick, Justin Lee Bonfiglio, Brandon Butler, Dwayne Buttler, Will Cross et al. 2020. Public Statement of Library Copyright Specialists: Fair Use E Emergency Remote Teaching E Research. Fecha de consulta: 20 de diciembre de 2020 .

https:/docs.google.com/document/d/10baTITJbFRh7D6dHVVvfgiGP2zqaMvm0EHHZYf2cBRk/mobilebasic

Armocida, Benedetta, Beatrice Formenti, Silvia Ussai, Francesca Palestra y Eduardo Missoni. 2020. "The Italian health system and the COVID-19 challenge". The Lancet Public Health 5 (5): e253. https://doi.org/10.1016/S2468-2667(20)30074-8

Bassel Khartabil Fellowship. 2020. Bassel Khartabil Fellowship Awarded to Dr. Tarek Loubani and Glia to Combat COVID-19. Fecha de consulta: 20 de diciembre de 2020. https://basselkhartabil.org/2020/04/02/second-fellowship-awarded.html

Broadband Commission for Sustainable Development. 2020. Broadband Commission Agenda for Action for Faster and Better Recovery. Fecha de consulta: 18 de diciembre de 2020 https://broadbandcommission.org/COVID19/Pages/default.aspx

CAS (Chinese Academy of Sciences). 2020. Scientific Literature Sharing Platform Built for COVID-19 Studies. Fecha de consulta: 18 de diciembre de 2020. http://ncov.cas.cn/en

CEPI (Coalition for Epidemic Preparedness Innovations). 2020. COVAX: CEPI's response to COVID-19-CEPI. Fecha de consulta: 20 de diciembre de 2020. https://cepi.net/COVAX/

Chakchouk, Moez y Stefania Giannini. 2020. Call for Joint Action: Supporting Learning and Knowledge Sharing through Open Educational Resources (OER). Paris: Unesco.

Chapman, Amy L. y Christine Greenhow. 2019. "Citizen-scholars: Social media and the changing nature of scholarship”. Publications 7 (1). https://doi.org/10.3390/publications7010011

Charmaz, Kathy. 2006. Constructing Grounded Theory. A Practical Guide Through Qualitative Analysis. London: SAGE Publications.

Choudhury, Suparna, Jennifer R. Fishman, Michelle L. McGowan y Eric T. Juengst. 2014. "Big data, open science and the brain: Lessons learned from genomics". Frontiers in Human Neuroscience 8 (mayo): 1-10. https://doi.org/10.3389/fnhum.2014.00239

Doshi, Peter. 2020. "Covid-19 vaccine trial protocols released”. The BMJ 371 (M4058): 1-2. https://doi.org/10.1136/bmj.m4058

EC (European Commission). 2011. "Horizon 2020 - The Framework Programme for Research and Innovation”, comunicado de prensa, Bruselas. https://eur-lex.europa.eu/legal-content/EN/TXT/PDF/?uri=CELEX:52011DC0808\&from $=\mathrm{EN}$ 
Elliott, Kevin C. y David B. Resnik. 2019. "Making open science work for science and society”. Environmental Health Perspectives 127 (7): 1-6. https://doi.org/10.1289/EHP4808

García-Peñalvo, Francisco J. 2010. "Open knowledge. Challenges and facts”. Open Information Review 34 (4): 520-539. https://doi.org/10.1108/14684521011072963

Hampton, Stephanie E., Sean S. Anderson, Sarah C. Bagby, Corinna Gries, Xueying Han, Edmund M. Hart, Matthew B. Jones et al. 2015. "The Tao of open science for ecology". Ecosphere 6 (7): art120. https://doi.org/10.1890/es14-00402.1

HEFCE (Higher Education Funding Council for England), RCUK (Research Councils UK), Universities UK (UUK) y Wellcome. 2016. Concordat On Open Research Data. https://www.ukri.org/wp-content/uploads/2020/10/UKRI-020920-ConcordatonOpenResearchData.pdf

Hick, John L. y Paul D. Biddinger. 2020. "Novel Coronavirus and Old Lessons Preparing the Health System for the Pandemic". New England Journal of Medicine 382 (20): e55. https://doi.org/10.1056/NEJMp2005118

Ibict (Instituto Brasileiro de Informação em Ciência e Tecnologia). 2020. Directorio de fuentes de información cientifica de acceso abierto sobre Coronavirus. Fecha de consulta: 20 de diciembre de 2020.

http://diretoriodefontes.ibict.br/coronavirus/index.php/es/ciencia-aberta-e-vida-espanol/

ILO (International Labour Organization). 2020. ILO Monitor: COVID-19 and the world of work. Sixth edition. Updated estimates and analysis. Ginebra: ILO.

Joshi, Amit, Preeti Bhaskar y Puneet Kumar Gupta. 2020. "Indian economy amid COVID-19 lockdown: A Perspective". Journal of Pure and Applied Microbiology 14 (mayo): 957-961. https://doi.org/10.22207/JPAM.14.SPL1.33

Mandal, Hasan. 2020. "Mobilizing the research ecosystem for scientific advances towards positive impact in thcontext of the COVID-19 pandemic". Turkish Journal of Medical Sciences 50 (SI-1): 485-488. https://doi.org/10.3906/sag-2004-180

McKiernan, Erin C., Philip E. Bourne, C. Titus Brown, Stuart Buck, Amye Kenall, Jennifer Lin, Damon McDougall et al. 2016. "How open science helps researchers succeed". eLife 5 (July): 1-19. https://doi.org/10.7554/eLife.16800

MIDAS (Models of Infectious Disease Agent Study). 2020. Online Portal for COVID-19 Modeling Research. Fecha de consulta: 19 de diciembre de 2020. https://midasnetwork.us/covid-19/

MOBS Lab (Laboratory for the Modeling of Biological + Socio-technical Systems). 2020. Analysis of the COVID-19 Epidemic. Fecha de consulta: 20 de diciembre de 2020. https://www.mobs-lab.org/2019ncov.html

NCBI (National Center for Biotechnology Information) y NLM (National Library of Medicine). 2020. Public Health Emergency COVID-19 Initiative. Fecha de consulta: 18 de diciembre de 2020 . https://www.ncbi.nlm.nih.gov/pmc/about/covid-19/?cmp=1 
NSF (National Science Foundation). 2015. Today's Data, Tomorrow's Discoveries. Increasing Access to the Results of Research Funded by the National Science Foundation. https://www.nsf.gov/pubs/2015/nsf15052/nsf15052.pdf

OECD (Organisation for Economic Cooperation and Development). 2007. OECD Principles and Guidelines for Access to Research Data From Public Funding. Paris: OECD Publications.

https://www.oecd.org/sti/inno/38500813.pdf\#: :text=The\%20OECD\%20Recommendation $\% 20$ states $\% 20$ that $\% 20$ member $\% 20$ countries $\% 20$ should,with\%20the \%20intention \%20 of\%20further\%20fostering $\% 20$ international $\% 20$ co-operation

OECD. 2015. "Making Open Science a Reality". OECD Science, Technology and Industry Policy Papers 25. Paris: OECD Publishing. https://doi.org/10.1787/5jrs2f963zs1-en

OECD. Secretary-General. 2020. Why open science is critical to combatting COVID-19. Paris: OECD. https://read.oecd-ilibrary.org/view/?ref=129_129916-31pgjnl6cb\&title=Why-open-science-is-critical-to-combatting-COVID-19

Olivé, León. 2007. La ciencia y la tecnología en la sociedad del conocimiento. Ética, política y epistemología. Ciudad de México: Fondo de Cultura Económica.

Peters, Michael A. 2010. "Three forms of the knowledge economy: Learning, creativity and openness". British Journal of Educational Studies 58 (1): 67-88. https://doi.org/10.1080/00071000903516452

Pfenninger, Stefan, Joseph DeCarolis, Lion Hirth, Sylvain Quoilin y Iain Staffell. 2017. "The importance of open data and software: Is energy research lagging behind?" Energy Policy 101 (febrero): 211-215. https://doi.org/10.1016/j.enpol.2016.11.046

RDA (Research Data Alliance). 2020. RDA COVID19 Case Statement. Fecha de consulta: 19 de diciembre de 2020.

https://www.rd-alliance.org/group/rda-covid19-rda-covid19-omics-rda-covid19-epidemiology-rda-covid19-clinical-rda-covid19-social

Shadmi, Efrat, Yingyao Chen, Inês Dourado, Inbal Faran-Perach, John Furler, Peter Hangoma, Piya Hanvoravongchai et al. 2020. "Health equity and COVID-19: global perspectives". International Journal for Equity in Health 19 (1): 104. https://doi.org/10.1186/s12939-020-01218-z

Taskinsoy, John. 2020. "Cost Implications of the Great Lockdown due to the Novel Coronavirus Outbreak”. SSRN Electronic Journal, 18 de mayo. https://doi.org/10.2139/ssrn.3604573

Unesco (United Nations Educational, Scientific and Cultural Organization). 2017a. Libre acceso a la información científica. Fecha de consulta: 12 de diciembre de 2020. http://www.unesco.org/new/es/communication-and-information/access-to-knowledge/open-access-to-scientific-information/

Unesco. 2017b. Overview of OA in Latin America and the Caribbean. Fecha de consulta: 13 de diciembre de 2020 . http://www.unesco.org/new/en/communication-and-information/portals-and-platforms/goap/access-by-region/latin-america-and-the-caribbean/

Unesco. 2020a. COVID-19 Pandemic: Language matters. Fecha de consulta: 18 de diciembre de 2020. https://en.iyil2019.org/all-resources/covid-19-pandemic-language-matters/ 
Unesco. 2020b. Open access to facilitate research and information on COVID-19. Fecha de consulta: 5 de diciembre de 2020.

https://en.unesco.org/covid19/communicationinformationresponse/opensolutions

Van Schalkwyk, May C. I., Thomas R. Hird, Nason Maani, Mark Petticrew y Anna B. Gilmore. 2020. “The perils of preprints”. The BMJ 370 (m3111): 1-2. https://doi.org/10.1136/bmj.m3111

Vicente-Saez, Ruben y Clara Martinez-Fuentes. 2018. "Open Science now: A systematic literature review for an integrated definition”. Journal of Business Research 88 (January): 428-436. https://doi.org/10.1016/j.jbusres.2017.12.043

Wellcome. 2020. "Sharing research data and findings relevant to the novel coronavirus (COVID-19) outbreak”, comunicado de prensa, 30 de enero de 2020. https://wellcome.org/coronavirus-covid-19/open-data

White, Marilyn Domas y Emily E. Marsh. 2006. "Content analysis: A flexible methodology". Library Trends 55 (1): 22-45. https://doi.org/10.1353/lib.2006.0053

Whyte, Angus y Graham Pryor. 2011. "Open Science in Practice: Researcher Perspectives and Participation”. International Journal of Digital Curation 6 (1): 199-213. https://doi.org/10.2218/ijdc.v6i1.182

World Bank. 2020. Global Economic Prospects. Washington DC. https://doi.org/10.1596/978-1-4648-1553-9

Zastrow, Mark. 2020. "Open science takes on the coronavirus pandemic". Nature 581 (7806): 109-110. https://doi.org/10.1038/d41586-020-01246-3

Para citar este texto:

Díaz Fragoso, Omar, Gabriela María Luisa Riquelme Alcantar y Gibrán Rivera González. 2021. "Ciencia Abierta y su papel durante la pandemia de COVID-19”. Investigación Bibliotecológica: archivonomía, bibliotecología e información 35 (88): 147-160.

http://dx.doi.org/10.22201/iibi.24488321xe.2021.88.58403 